\title{
SARS-CoV-2 viral load and antibody responses: the case for convalescent plasma therapy
}

\author{
Arturo Casadevall, ${ }^{1}$ Michael J. Joyner, ${ }^{2}$ and Liise-anne Pirofski ${ }^{3}$ \\ 'Department of Molecular Microbiology and Immunology, Johns Hopkins School of Public Health, Baltimore, Maryland, USA. ${ }^{2}$ Department of Anesthesiology and Perioperative Medicine, Mayo Clinic, \\ Rochester, Minnesota, USA. ${ }^{3}$ Division of Infectious Diseases, Albert Einstein College of Medicine and Montefiore Medical Center, Bronx, New York, USA.
}

\begin{abstract}
Most patients with COVID-19 lack antibody to SARS-CoV-2 in the first 10 days of illness while the virus drives disease pathogenesis. SARS-CoV-2 antibody deficiency in the setting of a tissue viral burden suggests that using an antibody as a therapeutic agent would augment the antiviral immune response. In this issue of the $J C I$, Wang and collaborators describe the kinetics of viral load and the antibody responses of 23 individuals with COVID-19 experiencing mild and severe disease. The researchers found that (a) individuals with mild and severe disease produced neutralizing IgG to SARS-CoV-2 10 days after disease onset, (b) SARS-CoV-2 persisted longer in those with severe disease, and $(c)$ there was cross-reactivity between antibodies to SARS-CoV-1 and SARS-CoV-2, but only antibodies from patients with COVID-19 neutralized SARS-CoV-2. These observations provide important information on the serological response to SARSCoV-2 of hospitalized patients with COVID-19 that can inform the use of convalescent plasma therapy.
\end{abstract}

the course of disease (11). This was based on the correlation between the appearance of specific serum antibody and clinical improvement in patients who recovered. Hence, the administration of convalescent plasma in the period before a patient develops their own antibody to SARS-CoV-2 may provide ready-made antibodies that mediate antiviral activity and augment the developing immune response.

Antibodies may mediate antiviral effects through numerous mechanisms, including direct antimicrobial effects such as viral neutralization as well as antibody-dependent cellular cytotoxicity (ADCC) and modulation of the inflammatory response (12). In fact, convalescent plasma therapy in patients with COVID-19, including those with severe disease, was shown to mediate viral clearance in several case studies and in a randomized controlled trial in which it led to rapid viral clearance from infected tissues $(2,13,14)$.

The observation that patients with severe COVID-19 disease produced higher antibody titers than patients with mild disease is consistent with what is described in other diseases such as tuberculosis, where symptomatic patients have higher titer responses (15). Although some may question the functional significance of high antibody levels in individuals with severe disease and this may raise the concern of detrimental antibody effects, such as antibody-dependent enhancement (16), we caution against such interpretations. Instead, the generation of more antibody is likely to reflect the strong inflammatory response associated with severe disease that contributes to the pathogenesis of COVID-19 (17).

\section{An exuberant inflammatory response}

Consistent with the observation that severe COVID-19 produces higher antibody titers, in this issue of the JCI, Wang et al. 


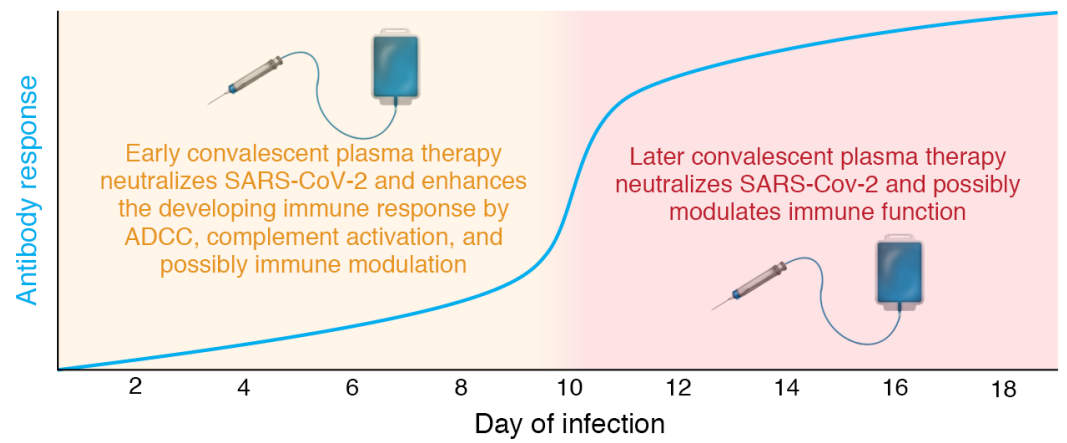

Figure 1. Early and later administration of convalescent plasma in the context of the developing antibody response. Patients with COVID-19 mount measurable antibody responses around day 10 , which peaks by day 15 , suggesting that convalescent plasma may benefit patients early in the disease course.

found that IgM was more likely to be present in the plasma of individuals with severe disease (18), which may reflect increased B cell recruitment in the setting of a more exuberant inflammatory response. In addition, as patients with severe disease had a higher and more persistent tissue viral burden than those with mild disease, stronger antibody responses may correlate with the viral burden. In our view, the relationship between higher antibody levels and severe disease may reflect host damage stemming from the inflammatory response to SARSCoV-2, rather than a detrimental effect of antibody. Indeed, convalescent plasma administration to thousands of patients with COVID-19 showed a safety profile comparable to regular plasma, implying that any untoward effects from the presence of specific antibody to SARS-CoV-2, such as antibody-dependent enhancement or the triggering of cytokine storm, were nil or rare (2).

The fact that most patients had strong antibody responses to SARS-CoV-2 by day 15 in Wang et al. raises the question of whether additional antibodies from the infusion of convalescent plasma are likely to have any effect after the second week of disease (18). Although this question cannot be answered without clinical trials, it is possible that even patients with late-stage disease may benefit from convalescent plasma if the quality of antibody responses during active disease and convalescence differ. We know that it takes time for antibodies to affinity mature, a process that is likely to imbue them with more effective antiviral properties. Convalescent plasma promotes viral clearance, even in severely ill patients, even when given more than two weeks after COVID-19 onset (19), which is when the Wang et al. study suggests endogenous antibody responses are present (18). This late viral clearance hints that early and convalescent antibody responses may differ in efficacy (Figure 1). Similarly, it is noteworthy that in the Wang et al. study (18), patients who were severely ill with COVID-19 continued to shed virus despite having neutralizing antibodies. Given that a hallmark of COVID-19 is lymphopenia, isotype switching and affinity maturation, which depend on $\mathrm{T}$ cell help, may be impaired in this disease. Hence, future studies should investigate the quality of antibodies to SARS-CoV-2 as a function of time and correlate antibody characteristics such as isotype and affinity with their efficacy in reducing viral shedding.

The findings of the Wang et al. study are also relevant for the selection of convalescent plasma donors (18). The fact that individuals with severe disease had higher antibody titers than those with mild disease parallels a study from Korea showing lower IgG amounts in individuals with asymptomatic and mild disease (20). If convalescent plasma antibody titer correlates with efficacy, then individuals who have recovered from severe disease may make better donors. Interestingly, Wang et al. show that sera from patients with severe disease had measurable IgM, whereas that of patients with mild disease did not (18). IgM is essential for protection against West Nile virus and influenza in experimental models $(21,22)$. Whether IgM plays a role in convalescent plasma therapy for COVID-19 is import- ant because next-generation antibody preparations such as hyperimmune globulin do not include IgM, and most monoclonal antibodies are engineered as IgGs. Since there is evidence that the antibody response to COVID-19 correlates positively and quantitatively with disease severity, providing enough antibody at the right time may avert severe disease by limiting viral proliferation and the ensuing inflammatory response. However, in addition to determining the quantity of antibody that is necessary to mediate a beneficial effect, it is important that future studies also establish the qualitative characteristics that contribute to convalescent plasma efficacy.

\section{Conclusion}

In summary, the description of the viral and antibody response kinetics in patients with COVID-19 by Wang et al. (18) reinforces the case for early convalescent plasma therapy. Given that the study shows that antibody is absent in the first 10 days of illness, providing an amount of antibody that can induce viral clearance during this time may overcome this early antibody deficiency. The fact that these patients have tissue viral burdens that are likely to drive the inflammatory response further supports the use of convalescent plasma therapy to promote viral clearance $(2,13,23,24)$. It is also possible that administration of antibody to patients early in disease may capitalize on the ability of some antibodies to function as immune modulators (25) and focus the immune response on determinants that are not prominent in the natural response. Future studies should also investigate the immune modulating properties of specific antibodies to SARS-CoV-2.

\section{Acknowledgments}

AC was supported in part by NIH grants AI052733, AI15207, and HL059842. MJL was supported by NIH grant R35, NHLBI grant 139854, and Biomedical Advanced Research and Development Authority (BARDA) grant 75A50120C00096. LP was supported in part by NIH grants AI123664 and AI143453 and a grant from the Mathers Foundation.

Address correspondence to: Arturo Casadevall, Department of Molecular Microbiology and Immunology, Johns Hopkins 
School of Public Health, Room E5132, 615 North Wolfe Street, Baltimore, Maryland 21205, USA. Phone: 410.955.3457; Email: acasade1@jhu.edu.

1. Wang Y, et al. Kinetics of viral load and antibody response in relation to COVID-19 severity. J Clin Invest. 2020;130(10):5235-5244.

2. Joyner MJ, et al. Early safety indicators of COVID-19 convalescent plasma in 5000 patients. J Clin Invest. 2020;130(9):4791-4797.

3. Li L, et al. Effect of convalescent plasma therapy on time to clinical improvement in patients with severe and life-threatening COVID-19: A randomized clinical trial [published online June 3, 2020]. Jama. https://doi.org/10.1001/ jama.2020.10044.

4. Hegerova L, et al. Use of convalescent plasma in hospitalized patients with Covid-19 - case series [published online June 19, 2020]. Blood. https:// doi.org/10.1182/blood.2020006964.

5. Xia X, et al. Improved clinical symptoms and mortality among patients with severe or critical COVID-19 after convalescent plasma transfusion [published online June 22, 2020]. Blood. https://doi.org/10.1182/blood.2020007079.

6. Casadevall A, Pirofski LA. The convalescent sera option for containing COVID-19. J Clin Invest. 2020;130(4):1545-1548.

7. Bloch EM, et al. Deployment of convalescent plasma for the prevention and treatment of COVID-19. J Clin Invest. 2020;130(6):2757-2765.

8. Luke TC, Casadevall A, Watowich SJ, Hoffman SL, Beigel JH, Burgess TH. Hark back: passive immu- notherapy for influenza and other serious infections. Crit Care Med. 2010;38(4 Suppl):e66-e73.

9. Hung IF, et al. Convalescent plasma treatment reduced mortality in patients with severe pandemic influenza A (H1N1) 2009 virus infection. Clin Infect Dis. 2011;52(4):447-456.

10. Cheng Y, et al. Use of convalescent plasma therapy in SARS patients in Hong Kong. Eur J Clin Microbiol Infect Dis. 2005;24(1):44-46.

11. Flexner S. The results of the serum treatment in thirteen hundred cases of epidemic meningitis. JExp Med.1913;17(5):553-576.

12. Cecil RL. Effects of early serum treatment on pneumococcus Type I pneumonia. Trans Am Clin Climatol Assoc. 1936;52:52-63.

13. Casadevall A, Pirofski LA. A new synthesis for antibody-mediated immunity. Nat Immunol. 2011;13(1):21-28.

14. Duan K, et al. Effectiveness of convalescent plasma therapy in severe COVID-19 patients. Proc Natl Acad Sci USA. 2020;117(17):9490-9496.

15. Achkar JM, et al. Antibodies against immunodominant antigens of Mycobacterium tuberculosis in subjects with suspected tuberculosis in the United States compared by HIV status. Clin Vaccine Immunol. 2010;17(3):384-392.

16. Yu X, Prados-Rosales R, Jenny-Avital ER, Sosa K, Casadevall A, Achkar JM. Comparative evaluation of profiles of antibodies to mycobacterial capsular polysaccharides in tuberculosis patients and controls stratified by HIV status. Clin Vaccine Immunol. 2012;19(2):198-208.

17. Wan Y, et al. Molecular mechanism for antibody-dependent enhancement of coronavirus entry. J Virol. 2020;94(5):e02015-19.
18. Pirofski LA, Casadevall A. Pathogenesis of COVID-19 from the perspective of the damage-response framework. mBio. 2020;11(4):e01175-20.

19. Duan SM, et al. Stability of SARS coronavirus in human specimens and environment and its sensitivity to heating and UV irradiation. Biomed Environ Sci. 2003;16(3):246-255.

20. Choe PG, et al. Antibody responses to SARS-CoV-2 at 8 weeks postinfection in asymptomatic patients. Emerging Infect Dis. 2020;26(10):eid2610.202211.

21. Diamond MS, Sitati EM, Friend LD, Higgs S Shrestha B, Engle M. A critical role for induced IgM in the protection against West Nile virus infection. J Exp Med. 2003;198(12):1853-1862.

22. Baumgarth N, Herman OC, Jager GC, Brown LE, Herzenberg LA, Chen J. B-1 and B-2 cell-derived immunoglobulin $\mathrm{M}$ antibodies are nonredundant components of the protective response to influenza virus infection. J Exp Med. 2000;192(2):271-280

23. Zeng QL, et al. Effect of convalescent plasma therapy on viral shedding and survival in patients with coronavirus disease 2019. J Infect Dis. 2020;222(1):38-43.

24. Magleby R, et al. Impact of SARS-CoV-2 viral load on risk of intubation and mortality among hospitalized patients with coronavirus disease 2019 [published online June 30, 2020]. Clin Infect Dis. https://doi.org/10.1093/cid/ciaa851.

25. Brady LJ. Antibody-mediated immunomodulation: a strategy to improve host responses against microbial antigens. Infect Immun. 2005;73(2):671-678. 